\title{
Discriminating imaging findings of acute osteoporotic vertebral fracture: a prospective multicenter cohort study
}

\author{
Khalid Mohammad Qasem', Akinobu Suzuki ${ }^{*}$, Kentaro Yamada ${ }^{1}$, Masatoshi Hoshino², Tadao Tsujio²,
} Shinji Takahashi ${ }^{1}$ and Hiroaki Nakamura ${ }^{1}$

\begin{abstract}
Background: Appropriate treatment of osteoporotic vertebral fractures (OVF) requires knowledge of the age of the fracture. Although diagnostic imaging has made remarkable progress in recent years, it remains difficult to differentiate acute fractures from old. Our purpose was to investigate chronological changes in radiological findings after OVF and to identify discriminators of acute versus older injuries.

Methods: We evaluated 139 vertebrae in 136 patients. All patients underwent X-ray and magnetic resonance imaging (MRI) examination within 2 weeks of injury and again after 6 months. The anterior vertebral height ratio (AVHR) was calculated on lateral X-ray, and the intensity change of the posterior wall of the fractured vertebra was evaluated on T1-weighted MRI. The cutoff AVHR value to diagnose acute fracture was determined by receiver operating characteristic (ROC) curve analysis.

Results: Average AVHR fell from $84.6 \%$ at initial visit to $63.7 \%$ at 6 months. When acute fracture was defined as AVHR $>75 \%$, sensitivity was $85.6 \%$, specificity was $67.6 \%$, and positive predictive value was $72.6 \%$. On MRI, $83.5 \%$ of fractured vertebrae showed intensity change in the posterior wall in the acute stage, which fell to $41.7 \%$ of vertebrae after 6 months. When intensity change in the posterior wall and AVHR $>75 \%$ were both present, the specificity and positive predictive value for diagnosing acute fracture improved to $87.1 \%$ and $84.7 \%$, respectively.

Conclusions: This study suggests that vertebral fracture rarely shows significant collapse on X-ray in the first 2 weeks after injury. The combination of intensity change in the posterior wall on MRI and AVHR $>75 \%$ on X-ray indicates a high probability of acute fracture.
\end{abstract}

Keywords: Osteoporosis, Vertebral fracture, Plain X-ray, Magnetic resonance imaging, Chronological change

\section{Background}

The percentage of the population over 65 years of age is increasing in developed countries. In line with this trend, the number of patients with osteoporosis is also increasing $[1,2]$. The most common type of fracture associated with osteoporosis is osteoporotic vertebral fracture (OVF). Because OVF has a large adverse effect on activities of daily living and quality of life [3,4], appropriate treatment is essential at each stage of injury. In the early stages, conservative treatment is often used. Bracing or

\footnotetext{
* Correspondence: a-suzuki@msic.med.osaka-cu.ac.jp

'Department of Orthopaedic Surgery, Osaka City University Graduate School of Medicine, 1-4-3 Asahi-machi, Abeno-ku, Osaka 545-8585, Japan Full list of author information is available at the end of the article
}

patient education for daily activity should be started immediately, because delaying these therapeutic interventions may slow healing or may lead to severe vertebral collapse, affecting the patient's future ability in activities of daily living and impairing quality of life [5-7]. Delayed union and pseudarthrosis after OVF can cause prolonged back pain and progressive collapse of the fractured vertebral body [8]. Kyphosis-induced worsening of truncal balance and collapse of vertebral bodies have been cited as major causes of decreased quality of life in elderly patients [7]. For cases resistant to conservative treatment, alternative options such as vertebroplasty, kyphoplasty, and/or other surgical treatments such as osteotomy may be applied to stabilize the fractured vertebrae [9] or to 
correct alignment [10]. Because the choice of treatment varies with the stage of the fracture, it is important to know the duration after injury to select the appropriate treatment option. OVF is usually diagnosed on plain $\mathrm{X}$-ray, but it is often difficult to distinguish acute vertebral injury on plain films because of various vertebral deformities, including old fractures. Many studies have shown the usefulness of MRI in conjunction with plain X-ray in diagnosing OVF [11-13]. However, few reports have described the time-dependent changes in imaging findings on both plain radiographs and MRI after OVF, and the distinguishing features of acute fracture remain unknown.

In this study, we followed 136 patients with OVF for 6 months and investigated radiological changes over time. The purpose of this study was to evaluate the changes in vertebral height ratio on X-ray and intensity change on T1-weighted MR images and to identify indicators of acute versus chronic vertebral fractures associated with osteoporosis.

\section{Methods}

\section{Patient selection}

Twenty-five institutions in the Osaka area of Japan participated in this prospective cohort study [14,15]. Patients older than 65 years with recent OVFs were enrolled and provided informed consent. All patients were examined with plain radiographs and MRI of the spine, and orthopedic surgeons at each institution diagnosed vertebral fracture based on acute back pain with abnormal radiological findings. Pathological fractures associated with tumors were excluded. The study was preapproved by the Ethical Committees for Clinical Research at the respective institutions. Between June 2005 and September 2007, a total of 485 patients were enrolled. At the time of enrollment, all patients were treated conservatively. Treatment decisions, including type of brace, duration of bracing, and medications, were made by individual doctors based on their experience. Among the 485 patients, 15 died, 11 were excluded because of other diseases (e.g., heart failure, cerebral infarction, pulmonary emphysema), and 39 were lost to follow-up. In all, 420 patients completed the 6-month follow-up (86.6\% follow-up rate).

In this study, we included only those patients whose injury date was identified by pain onset or specific known injury. Patients whose MRI was not examined within 2 weeks after injury, whose MRI was not examined at 6month follow-up, and/or whose image quality was poor were excluded. Patients with additional acute or chronic fracture of adjacent vertebrae were also excluded because this could complicate comparison of the shape and color of the targeted acutely injured vertebrae with adjacent vertebrae. Overall, 139 vertebrae in 136 patients (21 men and 126 women) were analyzed in this study.

\section{Imaging analysis}

Plain X-rays were taken with patients in the lateral decubitus position. We calculated the anterior vertebral height ratio (AVHR) as the anterior body height of the fractured vertebra divided by the height of the adjacent intact vertebral body (Figure 1) [14,15] at the time of enrollment and at 6-month follow-up. Non-union at 6 months was diagnosed if there was instability (change in shape with vertebral cleft) of the fractured vertebra between flexed and extended positions on plain radiographs.

Three experienced orthopedic surgeons who were not provided with patient information evaluated the MRI images. Change in signal intensity at the posterior wall of fractured vertebrae was evaluated on T1-weighted images (Figure $2 \mathrm{a}, \mathrm{b}$ ) and recorded as positive or negative. The three surgeons initially evaluated all MRI images independently. The evaluations were consistent for 201 of 278 images $(72.3 \%)$. For the remaining images without consensus, the three doctors discussed the cases and together determined the final evaluation. In this study, we did not include the evaluation of T2-weighted images because intensity on these images varies too much between patients to achieve agreement on the evaluation [12].

\section{Statistical analysis}

Data are presented as mean \pm SD. Sensitivities and specificities for the best cutoff value of AVHR to diagnose acute fractures versus old fractures were calculated with receiver operating characteristic (ROC) curve analysis using a computer software (SPSS version 19.0; IBM Co., NY, USA).

\section{Results}

Patient demographic data are shown in Table 1. The mean patient age was $75.9 \pm 6.6$ years (range, 65-91 years). Fractures were most common at L1 (35.3\%), followed by T12 (26.6\%) and L2 (10.8\%). All patients were treated conservatively during the follow-up period.

\section{Chronological change in anterior vertebral height ratio on plain X-ray}

On plain X-ray within 2 weeks after injury, the mean AVHR was $84.6 \pm 11.7 \%$. One hundred twenty-five vertebrae (90\%) with acute OVF had AVHR greater than 70\% (Figure 3a). At 6-month follow-up, the mean AVHR decreased to $63.7 \pm 11.7 \%$, and $65.2 \%$ of all vertebrae had AVHR less than 70\% (Figure 3a). By 6 months after injury, $77.7 \%$ of vertebral fractures had more than $10 \%$ further collapse than at initial evaluation, and $24.5 \%$ of vertebrae had collapsed more than 30\% further (Figure 3b).

\section{Chronological change in intensity at posterior wall on MRI}

On T1-weighted MRI, 116 vertebrae (83.5\%) showed a low-intensity change at the posterior wall of the fractured vertebra in the acute stage, and 58 (41.7\%) had a 


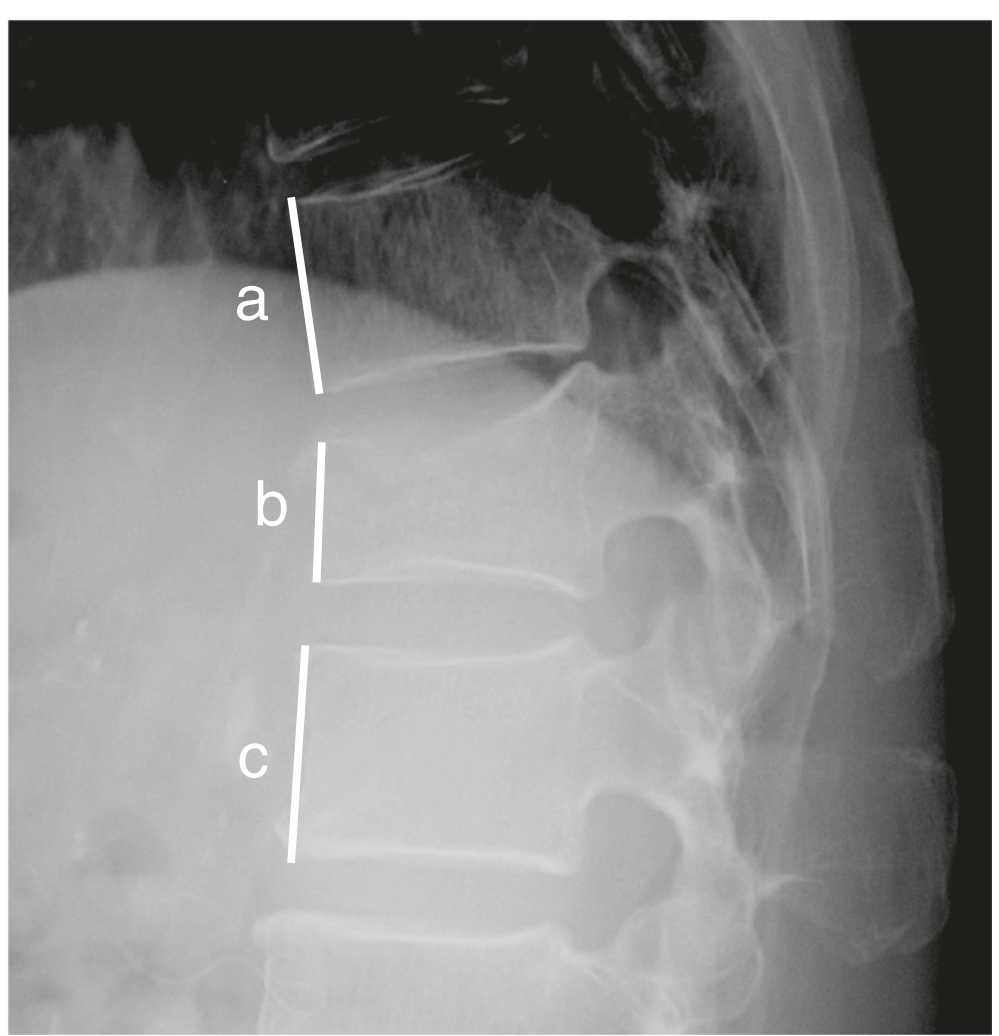

Figure 1 Anterior vertebral height ratio $=2 b /(a+c) \times 100(\%)$.

persistent hypointensity after 6 months. Among the vertebrae with low-intensity change in the acute stage, signal intensity had normalized by 6 months in 65 vertebrae (56\%).

\section{Sensitivity, specificity, and positive predictive value for} the diagnosis of acute OVF

The area under the curve (AUC) for the ROC (Figure 4) was 0.815 (95\% CI, 0.765-0.865), indicating good discrimination of acute OVF from older OVF based on the AVHR on plain X-ray. A cutoff value of $>75 \%$ for the AVHR gave optimal sensitivity and specificity for diagnosing acute versus older OVF (sensitivity 85.6\%, specificity 67.6\%, positive predictive value (PPV) 72.6\%, negative predictive value (NPV) $82.4 \%$ ). When acute fracture was diagnosed based on low-intensity change at the posterior wall on T1-weighted images, sensitivity was $83.5 \%$, specificity was $58.3 \%$, PPV was $66.7 \%$, and

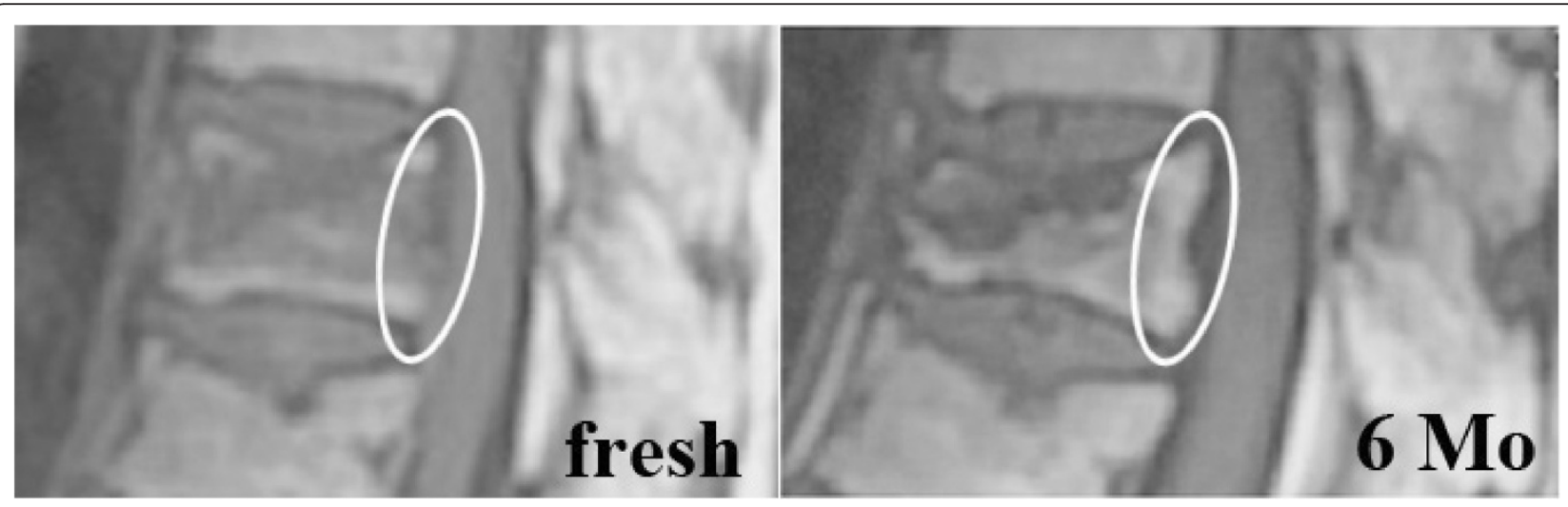

Figure 2 Representative image of chronological intensity change at posterior wall on T1-weighted magnetic resonance image. Intensity change at posterior wall was observed in the acute stage (left), but the intensity change was disappeared at 6 months (right). 
Table 1 Demographic data

\begin{tabular}{ll}
\hline Demographic data & Number \\
\hline Sex (female), $n$ (\%) & 116 (85.3) \\
Age, mean \pm SD, (range), years & $75.9 \pm 6.6$ (65-91) \\
Level, $n$ & \\
T6 & 1 \\
T7 & 1 \\
T8 & 1 \\
T9 & 5 \\
T10 & 1 \\
T11 & 10 \\
T12 & 37 \\
L1 & 49 \\
L2 & 15 \\
L3 & 11 \\
L4 & 8 \\
Period to first X-ray examination from injury, & $3.2 \pm 3.5$ (0-14) \\
mean \pm SD, (range), days & \\
Period to first MRI examination from injury, & $6.2 \pm 3.9$ (0-14) \\
mean \pm SD, (range), days & \\
Hospitalization, $n$ (\%) & $97(70.8)$ \\
Orthosis & \\
Tailor-made hard corsets, $n$ (\%) & $27(19.7)$ \\
Tailor-made elastic corsets, $n$ (\%) & $69(50.4)$ \\
Ready-made elastic corsets, $n$ (\%) & $26(19.0)$ \\
None & $14(10.2)$ \\
Non-union (6 months), $n$ (\%) & $15(10.8)$ \\
\hline & \\
\hline
\end{tabular}

NPV was $77.9 \%$. When both AVHR $>75 \%$ and intensity change were present, sensitivity decreased to $71.9 \%$ and NPV to $75.7 \%$, but the specificity and PPV markedly improved to $87.1 \%$ and $84.7 \%$, respectively.

\section{The relationship between posterior wall hypointensity and non-union at 6 months}

At 6-month follow-up, 14 vertebrae (10.1\%) were diagnosed with non-union. Among these, 7 did not show lowintensity change at the posterior wall on T1-weighted images at 6 months, although some intensity change was found on other parts of the vertebra or on the T2weighted image. Using low-intensity change at the posterior wall on T1-weighted images to diagnose non-union at 6 months, sensitivity, specificity, and positive predictive value were $50.0 \%, 59.2 \%$, and $12.1 \%$, respectively.

\section{Discussion}

In this study, we included only patients who underwent imaging studies within 2 weeks after injury. We attempted to clarify the characteristics of acute vertebral fracture that differentiate it from older fractures by comparing radiological images from the first 2 weeks with those obtained 6 months after injury. Several studies have focused on the diagnosis of OVF, but many of these aimed to differentiate acute OVF from malignant fracture or osteomyelitis [11,16,17]. To our knowledge, this is the first study to focus on the differential diagnosis of acute versus old fracture.

Because X-ray is the simplest and most common test used to diagnose OVF, many studies have been performed to establish diagnostic methods using plain films $[18,19]$. Genant et al. reported a semi-quantitative method that assessed OVF by visual determination of the extent of vertebral height reduction and morphological change [18]. This semi-quantitative method standardizes the evaluation of apparent change in vertebral dimensions, so that a vertebral fracture is identified if vertebral height is more than $20 \%-25 \%$ less than expected. This assessment is intelligible and easy to use; however, this and other quantitative methods may not be sufficient in diagnosing acute fractures. Pham et al. reported that 21 vertebral fractures in 16 patients presented with a typical history of acute back pain in an individual with osteoporosis [20]; however, substantial deformation of the vertebral body was not found on initial plain radiographs. Kanchiku et al. also reported that 10 out of 95 OVFs showing signal intensity changes on MRI were difficult to identify on plain radiographs because of almost complete lack of vertebral body collapse [12]. In the present study, $70.5 \%$ of the fractured vertebrae had anterior vertebral body height more than $80 \%$ of that expected in the first 2 weeks after injury, whereas the percentage decreased to $25.9 \%$ at 6 months. Along with the reports of Pham et al. [20] and Kanchiku et al. [12], the present results suggest that diagnosing acute fracture on X-ray is often difficult because of the lack of deformity in the acute stage.

MRI is considered a more accurate tool for OVF diagnosis than plain X-ray. Kanchiku et al. reported a diagnostic rate of $98 \%$ for fractured vertebral bodies by MRI, which was higher than the $87 \%$ by plain radiography [12]. Several abnormal MRI findings have been identified as typical signs of OVF: fracture line as low-intensity signal band on T1 image [11], bone edema as signal intensity change on T1- and/or T2-weighted images [17,21], and fluid sign as focal or linear hyperintense change on T2-weighted or short $\mathrm{T} 1$ inversion recovery (STIR) image [13]. However, in most studies, the period from onset of injury until MRI examination was not uniform, and it remains unclear which abnormal findings indicate acute fracture. Although the chronological changes in abnormal findings have not been well studied, there have been a few anecdotal reports on this issue. Yamato et al. reported that the bone marrow intensity change on T1-weighted images restores earlier 
(a)

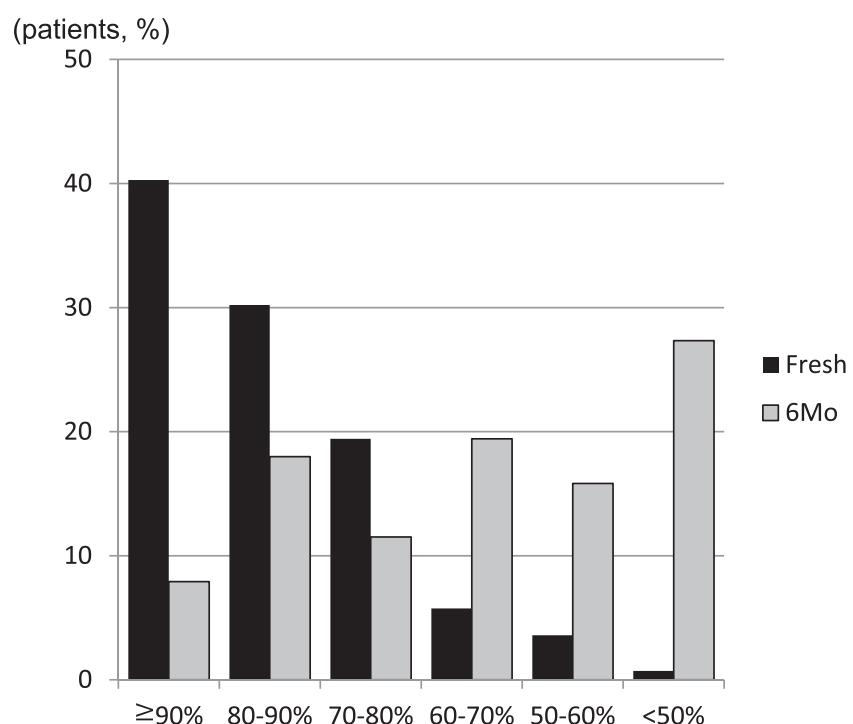

(AVHR) (b)

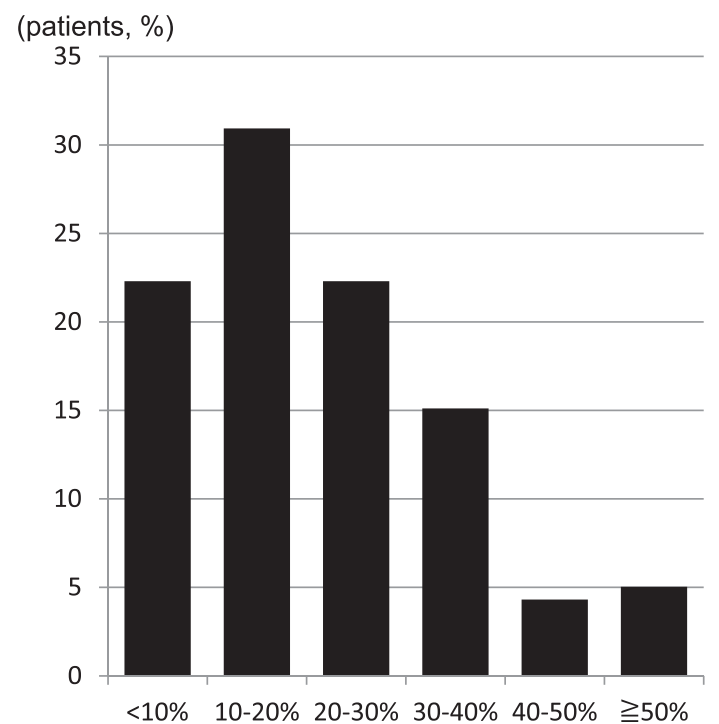

(AVHR)

Figure 3 Patient distribution of anterior vertebral height ratio (AVHR) on plain X-ray. (a) Patient distribution of AVHR within 2 weeks of injury (acute) and at 6 months after injury (old). (b) Change in AVHR between the two time points.

(a)

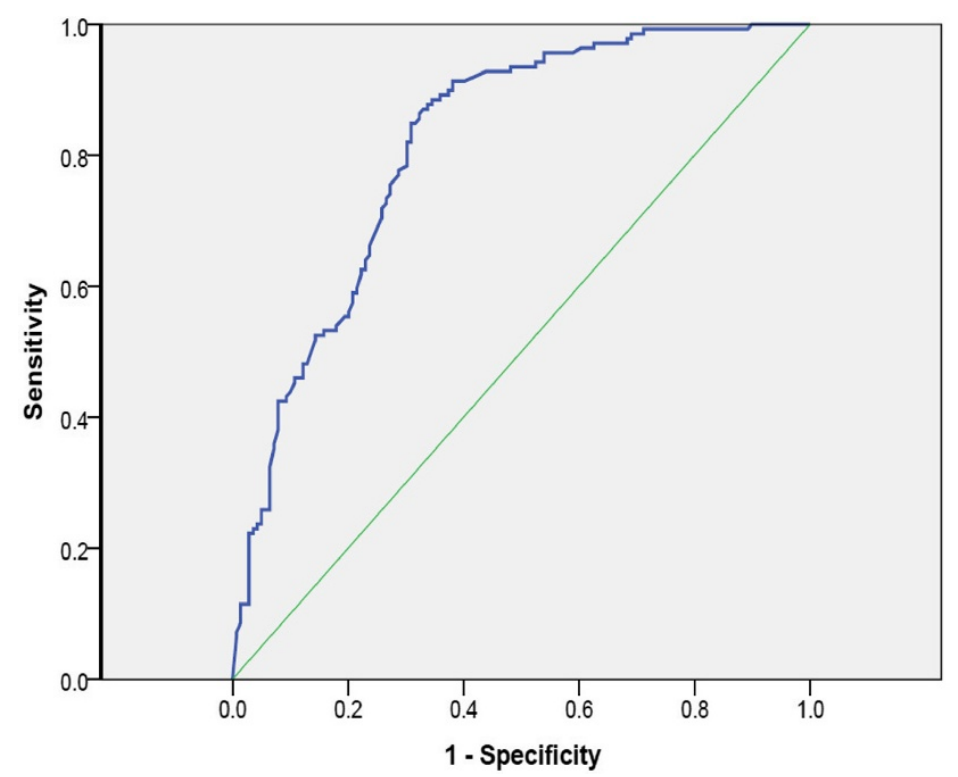

(b)

\begin{tabular}{|c|c|c|}
\hline Criterion & Sensitivity & Specificity \\
\hline$>100.0$ & 0 & 100 \\
95.1 & 21.6 & 97.1 \\
90.1 & 38.1 & 92.1 \\
85.2 & 52.5 & 84.9 \\
80.3 & 67.6 & 75.5 \\
75.2 & 84.9 & 68.3 \\
70.1 & 89.9 & 62.6 \\
65.3 & 92.8 & 52.5 \\
60.2 & 95.7 & 44.6 \\
55.3 & 97.1 & 36 \\
50.4 & 99.3 & 28.1 \\
45.3 & 99.3 & 19.4 \\
40.3 & 99.3 & 10.8 \\
35.1 & 100 & 5 \\
28.6 & 100 & 2.2 \\
24.0 & 100 & 1.4 \\
20.4 & 100 & 0.7 \\
$<17.0$ & 100 & 0 \\
\hline
\end{tabular}

Figure 4 Receiver operating characteristics (ROC) curve for discrimination of acute osteoporotic vertebral fracture from older fracture. (a) ROC curve for the discrimination of acute osteoporotic vertebral fracture from older fracture based on anterior vertebral collapse ratio, and (b) sensitivity and specificity for various cutoff criteria. 
than that on T2-weighted images [22]. Cho et al. demonstrated that restoration of signal intensity was slow near the endplate or the center of the vertebra in some types of fracture [23]. Based on these reports, we focused on intensity changes in the posterior wall of the fractured vertebral body on T1-weighted MRI to differentiate acute OVF.

The present study revealed that $75 \%$ is the best AVHR cutoff value to distinguish acute fracture from old and that this in combination with intensity change in the posterior wall on T1-weighted MRI is a good indicator of acute fracture. If a vertebral fracture is identified but the duration of the injury is uncertain, this combination of findings will be helpful to estimate the age of the fracture. However, the sensitivity and specificity of the abnormal intensity in the posterior wall on MRI were not high in diagnosing non-union, so normalization of intensity change in the posterior wall may not always indicate fracture healing. Further study including more detailed imaging analysis with longer follow-up will be necessary to identify the MRI findings that strongly support a diagnosis of non-union.

There are several limitations to note in this study. First, we only included patients whose date of fracture could be identified by the onset of pain or a specific injury. Therefore, these data may not be applicable for fractures without pain. Second, to simplify the study, we only evaluated AVHR on X-ray and posterior wall intensity change on T1-weighted MRI in the first 2 weeks after injury and 6 months after injury. Some vertebral fractures exhibit middle or posterior vertebral collapse without anterior collapse; in these cases, it may be more suitable to measure the middle or posterior vertebral height ratios rather than AVHR. Further studies that include middle/posterior vertebral height ratios, T2weighted images, and STIR images at more time points will provide more accurate discriminators for the diagnosis of fracture age and pseudarthrosis. Finally, we have to note that the sensitivity and specificity in this study are for discrimination between acute and older fractures, and not for differentiating acute vertebral fracture from other pathological processes. For the appropriate diagnosis of acute OVF, it is important to look for the presence of other abnormal findings on imaging studies that may indicate neoplastic vertebral fracture or osteomyelitis $[11,16,17]$.

\section{Conclusions}

The AVHR was high in the acute stage within 2 weeks of injury but significantly decreased by 6 months. On MRI, $42 \%$ of the fractured vertebrae with low signal intensity change in the posterior wall during the acute stage had normalized by 6 months after injury. The combination of intensity change in the posterior wall on
MRI and AVHR $>75 \%$ on X-ray indicates a high probability of acute fracture.

\section{Abbreviations}

OVF: osteoporotic vertebral fractures; MRI: magnetic resonance imaging; STIR: short T1 inversion recovery; AVHR: anterior vertebral height ratio; ROC: receiver operating characteristic; PPV: positive predictive value; NPV: negative predictive value.

\section{Competing interests}

The authors declare that they have no competing interests.

\section{Authors' contributions}

$\mathrm{KQ}$ participated in imaging analysis. AS participated in statistical analysis and helped draft the manuscript. KY participated in the design of the study and imaging analysis. $\mathrm{MH}$ and $\mathrm{TT}$ participated in data collection and data management. ST participated in statistical analysis. HN conceived of the study and participated in its design and coordination. All authors read and approved the final manuscript.

\section{Acknowledgements}

This research was supported by Japanese Health and Labor Sciences Research Grants for Comprehensive Research on Aging and Health. The authors also thank Dr. Hidetomi Terai, Dr. Hiromitsu Toyoda, Dr. Takashi Namikawa, Dr. Minori Kato, Dr. Akira Matsumura, Dr. Kazushi Takayama, Mrs. Masami Tatsumi, Mrs. Tomomi Tanaka, Ms. Tomoko Komaru, Ms. Aki Tanaka, Mrs. Asami Sato, and Mrs. Keiko Yoneda for their help in the collection of data and interviews with the patients. The authors also express sincere thanks to the doctors from Osaka City General Hospital, Osaka City Juso Hospital, Osaka City Kita Hospital, Osaka City Sumiyoshi Hospital, Kousaiin Hospital, Fujiidera Municipal Hospital, Izumi Municipal Hospital, Aeba Surgical Hospital, Yodogawa Christian Hospital, Ishikiri Seiki Hospital, Asakayama Hospital, Osaka Ekisaikai Hospital, Kyouwa Hospital, Saiseikai Nakatsu Hospital, Saiseikai Senri Hospital, Baba Memorial Hospital, Seikeikai Hospital, Nagayoshi Sougou Hospital, Nishinomiya Watanabe Hospital, Hankai Hospital, Higashisumiyoshi Morimoto Hospital, Shitennoji Hospital, Satou Hospital, and Tsujigeka Hospital for enrolling the patients in this prospective study.

\section{Author details}

${ }^{1}$ Department of Orthopaedic Surgery, Osaka City University Graduate School of Medicine, 1-4-3 Asahi-machi, Abeno-ku, Osaka 545-8585, Japan. ${ }^{2}$ Spine Center, Shiraniwa Hospital, 6-10-1 Shiraniwadai, Ikoma, Nara 630-0136, Japan.

Received: 24 August 2014 Accepted: 30 September 2014

Published online: 10 October 2014

\section{References}

1. Dawson-Hughes B, Looker AC, Tosteson AN, Johansson H, Kanis JA, Melton $\sqcup J$ 3rd: The potential impact of the National Osteoporosis Foundation guidance on treatment eligibility in the USA: an update in NHANES 2005-2008. Osteoporos Int 2012, 23:811-820.

2. Yoshimura N, Muraki S, Oka H, Mabuchi A, En-Yo Y, Yoshida M, Saika A, Yoshida H, Suzuki T, Yamamoto S, Ishibashi H, Kawaguchi H, Nakamura K, Akune T: Prevalence of knee osteoarthritis, lumbar spondylosis, and osteoporosis in Japanese men and women: the research on osteoarthritis/ osteoporosis against disability study. J Bone Miner Metab 2009, 27:620-628.

3. Center JR, Nguyen TV, Schneider D, Sambrook PN, Eisman JA: Mortality after all major types of osteoporotic fracture in men and women: an observational study. Lancet 1999, 353:878-882.

4. Suzuki N, Ogikubo O, Hansson T: Previous vertebral compression fractures add to the deterioration of the disability and quality of life after an acute compression fracture. European Spine Journal 2010, 19:567-574.

5. Kishikawa Y: Initial non-weight-bearing therapy is important for preventing vertebral body collapse in elderly patients with clinical vertebral fractures. Int J Gen Med 2012, 5:373-380.

6. Pfeifer M, Kohlwey L, Begerow B, Minne HW: Effects of two newly developed spinal orthoses on trunk muscle strength, posture, and quality-of-life in women with postmenopausal osteoporosis: a randomized trial. Am J Phys Med Rehabil 2011, 90:805-815.

7. Takahashi T, Ishida K, Hirose D, Nagano Y, Okumiya K, Nishinaga M, Matsubayashi K, Doi Y, Tani T, Yamamoto H: Trunk deformity is associated 
with a reduction in outdoor activities of daily living and life satisfaction in community-dwelling older people. Osteoporos Int 2005, 16:273-279.

8. Hoshino M, Nakamura H, Terai H, Tsujio T, Nabeta M, Namikawa T, Matsumura A, Suzuki A, Takayama K, Takaoka K: Factors affecting neurological deficits and intractable back pain in patients with insufficient bone union following osteoporotic vertebral fracture. Eur Spine J 2009, 18:1279-1286.

9. Heini PF: The current treatment-a survey of osteoporotic fracture treatment. Osteoporotic spine fractures: the spine surgeon's perspective. Osteoporos Int 2005, 16(Suppl 2):S85-S92.

10. Suk SI, Kim JH, Lee SM, Chung ER, Lee JH, Suk SI, Kim JH, Lee SM, Chung ER, Lee $\mathrm{JH}$ : Anterior-posterior surgery versus posterior closing wedge osteotomy in posttraumatic kyphosis with neurologic compromised osteoporotic fracture. Spine (Phila Pa 1976) 2003, 28:2170-2175

11. Abdel-Wanis ME, Solyman MT, Hasan NM: Sensitivity, specificity and accuracy of magnetic resonance imaging for differentiating vertebral compression fractures caused by malignancy, osteoporosis, and infections. J Orthop Surg (Hong Kong) 2011, 19:145-150.

12. Kanchiku T, Taguchi T, Kawai S: Magnetic resonance imaging diagnosis and new classification of the osteoporotic vertebral fracture. J Orthop Sci 2003, 8:463-466.

13. Yuh WT, Mayr NA, Petropoulou K, Beall DP: MR fluid sign in osteoporotic vertebral fracture. Radiology 2003, 227:905.

14. Matsumoto T, Hoshino M, Tsujio T, Terai H, Namikawa T, Matsumura A, Kato M, Toyoda H, Suzuki A, Takayama K, Takaoka K, Nakamura H: Prognostic factors for reduction of activities of daily living following osteoporotic vertebral fractures. Spine (Phila Pa 1976) 2012, 37:1115-1121.

15. Tsujio T, Nakamura $H$, Terai H, Hoshino M, Namikawa T, Matsumura A, Kato M, Suzuki A, Takayama K, Fukushima W, Kondo K, Hirota Y, Takaoka K: Characteristic radiographic or magnetic resonance images of fresh osteoporotic vertebral fractures predicting potential risk for nonunion: a prospective multicenter study. Spine (Phila Pa 1976) 2011, 36:1229-1235.

16. Vaccaro AR, Shah SH, Schweitzer ME, Rosenfeld JF, Cotler JM: MRI description of vertebral osteomyelitis, neoplasm, and compression fracture. Orthopedics 1999, 22:67-73. quiz 74-65.

17. Yuh WT, Zachar CK, Barloon TJ, Sato Y, Sickels WJ, Hawes DR: Vertebral compression fractures: distinction between benign and malignant causes with MR imaging. Radiology 1989, 172:215-218.

18. Genant HK, Wu CY, Van Kuijk C, Nevitt MC: Vertebral fracture assessment using a semiquantitative technique. J Bone Miner Res 1993, 8:1137-1148.

19. Jiang $G$, Eastell $R$, Barrington NA, Ferrar $L$ : Comparison of methods for the visual identification of prevalent vertebral fracture in osteoporosis. Osteoporos Int 2004, 15:887-896.

20. Pham T, Azulay-Parrado J, Champsaur P, Chagnaud C, Legre V, Lafforgue P: "Occult" osteoporotic vertebral fractures: vertebral body fractures without radiologic collapse. Spine (Phila Pa 1976) 2005, 30:2430-2435

21. Tan DY, Tsou IY, Chee TS: Differentiation of malignant vertebral collapse from osteoporotic and other benign causes using magnetic resonance imaging. Ann Acad Med Singapore 2002, 31:8-14.

22. Yamato M, Nishimura G, Kuramochi E, Saiki N, Fujioka M: MR appearance at different ages of osteoporotic compression fractures of the vertebrae. Radiat Med 1998, 16:329-334.

23. Cho T, Matsuda M, Sakurai M: MRI findings on healing process of vertebral fracture in osteoporosis. J Orthop Sci 1996, 1:16-33.

\section{Submit your next manuscript to BioMed Central and take full advantage of:}

- Convenient online submission

- Thorough peer review

- No space constraints or color figure charges

- Immediate publication on acceptance

- Inclusion in PubMed, CAS, Scopus and Google Scholar

- Research which is freely available for redistribution 